\title{
Terapia ocupacional e saúde mental: construindo lugares de inclusão social*
}

Occupational therapy and mental health: building places for social inclusion Terapia ocupacional y salud mental: construyendo lugares de inclusión social

\author{
Marli B. Santos Ribeiro \\ Luiz Roberto de Oliveira ${ }^{2}$
}

\section{Introdução}

O movimento de transformação da assistência psiquiátrica no Brasil

A tendência central da assistência aos loucos, no Brasil, desde seus primórdios, foi de excluí-los, num primeiro momento, junto com os desocupados, os inadaptados e perturbadores da ordem social nas santas casas, onde eles recebiam um tratamento diferente dos demais, amontoados nos porões, sem assistência médica, tendo seus sintomas reprimidos por espancamentos ou contenção em troncos, condenados à morte por maus tratos físicos, desnutrição e doenças infecciosas. Neste período, eles não se diferenciavam das outras categorias marginais pelos conceitos nosográficos ou psicopatológicos e sim, pelos critérios de razão e desrazão. Posteriormente, com o surgimento da psiquiatria, os mesmos passaram a ser colocados em hospícios e considerados doentes mentais (Resende, 1987).

Os primeiros hospícios vieram responder à demanda de organização das cidades, relacionada com o projeto ideal (valores morais, éticos e políticos) da sociedade moderna que estava se constituindo no país. A partir da inauguração do Hospício D. Pedro II, em 1852, houve uma constante necessidade de ampliação e solidificação deste equipamento como única forma de tratamento dos doentes mentais (Araújo, 1999).

Aos poucos a psiquiatria foi-se tornando científica, reconhecida como especialidade médica e foi avançando nos estudos anatomo-clínicos que relacionavam a doença com dano cerebral, passando-se a compreendê-la numa abordagem biológica (Silva Filho, 1987). Esta abordagem localiza fatores internos ao indivíduo como causa da doença, sendo eliminadas todas as variáveis externas que podem contribuir para o desenvolvimento da mesma. Nesta lógica, as propostas de intervenção são a administração de psicofármacos e a contenção dos sintomas por meio da internação psiquiátrica (Lussi, 2003).

O período da ditadura militar compreendido entre 1964 a 1985, provocou sérias

\footnotetext{
* Elaborado a partir de dissertação de Mestrado (Ribeiro, 2003), projeto financiado pela FAPESP (Proc. N. 2002/02101-4).

${ }^{1}$ Terapeuta ocupacional, Departamento de Neurologia e Psiquiatria, Faculdade de Medicina de Botucatu, Universidade Estadual Paulista, FMB-Unesp, Botucatu, SP. <marlisribeiro@ig.com.br>

${ }^{2}$ Professor, Departamento de Saúde Pública, Programa de Pós-Graduação em Saúde Coletiva, FMB-Unesp. <luizoliv@fmb.unesp.br>

${ }^{1}$ Rua Luiz Castelletti, 348

Distrito de Rubião Júnior - Botucatu, SP

$18.618-000$
} 


\section{ESPAÇO ABERTO}

transformações sociais, políticas e econômicas no país. Estas transformações levaram a uma política de privatização da assistência e, conseqüentemente, a um sucateamento da saúde pública, devido à falta de investimento do governo. $\mathrm{Na}$ assistência psiquiátrica optou-se pela contratação de leitos em hospitais privados que aumentaram rapidamente, pois os donos dos hospitais visavam obter lucro com as internações (Resende, 1987).

Enquanto os países europeus e os Estados Unidos vivenciavam um processo de transformação da assistência psiquiátrica com ênfase na desospitalização, os brasileiros assistiam a uma expansão dos leitos nos hospitais privados financiados pelo Estado. Para se ter uma idéia desta expansão, em vinte anos (de 1950 até 1970), a população brasileira cresceu $82 \%$ e a população dos hospitais psiquiátricos 213\% (Cezarino, 1989). Este aumento resultou no agravamento da situação dos hospitais psiquiátricos brasileiros, com superpopulação, deficiência de pessoal, maus tratos, péssimas condições de hotelaria e pouca utilidade para o tratamento.

No final da década de 1970, aproveitando o momento político de redemocratização do país, os profissionais que trabalhavam nesses hospitais, de maneira organizada, denunciaram a violência, ausência de recursos para a área, negligência, utilização da psiquiatria como instrumento técnico-científico de controle social e reivindicaram a criação de projetos alternativos ao modelo asilar (Amarante, 2000).

O movimento de reforma sanitária que teve início na década de 1970 foi importante para impulsionar as transformações na área de assistência psiquiátrica. Esse movimento, que se concretizou por meio da realização de conferências de saúde, tinha como lema: a saúde como direito de todos e dever do Estado, e baseava-se em princípios como regionalização, hierarquização, participação comunitária, integralidade e eqüidade (Yasui, 1999).

Em 1987 realizou-se a primeira conferência de saúde mental, a segunda foi em 1992 e a terceira conferência aconteceu em 2001, com expressiva participação dos usuários e familiares. Nesta conferência discutiu-se a necessidade da reorientação do modelo assistencial, por meio de eixos fundamentais como: controle social, acessiblidade e direitos, financiamento para a área e recursos humanos (Brasil, 2002).

Desde 1989 tramitava no Congresso Nacional uma lei de autoria do Deputado Paulo Delgado que só foi aprovada em 2001, após uma mobilização intensa dos trabalhadores da área, dos usuários, dos familiares e da mídia. Esta lei regulamenta os direitos do doente mental em relação ao tratamento, propõe a extinção progressiva dos manicômios, a substituição dos hospitais psiquiátricos por outros recursos assistenciais e regulamenta a internação psiquiátrica compulsória (Amarante, 2000).

Ao longo desses anos foi criada uma rede diferenciada e normalizada de serviços substitutivos ao hospital psiquiátrico: hospitais-dia, centros de atenção psicossocial, destacando-se o tipo III (com funcionamento 24 horas e aos finais de semana), leitos psiquiátricos em hospitais gerais, centros de convivência, oficinas terapêuticas, serviços residenciais terapêuticos, entre outros.

O processo de transformação da assistência psiquiátrica brasileira foi fortemente influenciado pelas experiências de reformas realizadas nos Estados Unidos e na Europa (Pitta, 1984; Amarante, 2000). 
O movimento de psiquiatria preventiva ou comunitária, que aconteceu nos Estados Unidos, no início na década de 1950, preconizava que o objeto da psiquiatria não devia ser a doença, mas, a saúde mental. Numa definição de Saraceno (2001, p.145), a saúde mental seria "a condição de saúde mental dos indivíduos e o conjunto de ações para promovê-la e mantê-la", passando-se "a considerar as dimensões psicológicas e sociais de saúde e os fatores psicossociais que determinam saúde e doença". Para o entendimento da doença buscaram-se outras áreas de conhecimento, como a psicologia, a sociologia e a antropologia, retirando-se a exclusividade da medicina (Amarante, 2000).

Do continente europeu as influências vieram da França, da Inglaterra e, sobretudo, do movimento da psiquiatria democrática italiana. Esta experiência, que começou na década de 1960, teve como princípio básico romper com a lógica segregativa e violenta da instituição psiquiátrica e transformar as relações entre doente, médico, equipe hospitalar e sociedade (Basaglia, 1991). Propunha o deslocamento da clinica centrada na doença para a invenção de cuidados dirigidos à pessoa doente, a desconstrução do hospital psiquiátrico e a criação de serviços substitutivos, em que se respeitasse o direito social e aumentasse o poder contratual dos usuários (Mângia, 2002).

\section{A atuação da terapia ocupacional diante da transformação da assistência psiquiátrica}

A profissão terapia ocupacional foi criada no início do século XX, nos Estados Unidos. Teve sua prática reconhecida no contexto da reabilitação física e mental pela necessidade de reinserir os traumatizados de guerra na sociedade (Benetton, 1991).

No Brasil, a profissão foi criada em 1959. Na área de psiquiatria tinha sua prática voltada a assistência hospitalocêntrica, com a tarefa de ocupar os pacientes, num processo de manutenção e organização dos hospitais e de reabilitação, tendo em vista que, com o advento das terapêuticas biológicas e farmacológicas, os pacientes melhoravam rapidamente dos sintomas (Benetton, 1991).

Diante das transformações na assistência psiquiátrica, esta profissão vem buscando uma legitimidade enquanto área de atuação e de produção de saber. Para tanto, os terapeutas ocupacionais têm procurado aprimorar-se teorica, tecnica e politicamente para a atuação na rede de serviços substitutivos, em nível de prevenção e promoção de saúde, tratamento, reabilitação e inclusão social.

No final da década de 1970, algumas terapeutas ocupacionais defendendo a função terapêutica, incorporam conceitos psicodinâmicos baseados na psicanálise e na psicologia e criam um método de tratamento. Neste método, a terapia ocupacional é definida por uma dinâmica relacional entre terapeuta-paciente-atividade em que se compõe uma trilha associativa num campo transferencial (Benetton, 1991). Este método tem sido amplamente divulgado entre os terapeutas ocupacionais e aplicado em pacientes com transtornos mentais graves, com o objetivo de manter a saúde mental e a sociabilidade.

A experiência da desinstitucionalização italiana trouxe inovações para a terapia ocupacional no campo da reforma psiquiátrica, ao assumir como objeto da ação terapêutica a pessoa e suas necessidades e não a doença e os sintomas. Neste contexto, a ação terapêutica deve investir na complexidade da vida cotidiana da pessoa, englobando os aspectos: práticos, concretos, simbólicos, relacionais e materiais, de forma a produzir movimentos capazes de oferecer suportes, proteção e resolução de 


\section{ESPAÇO ABERTO}

problemas que contribuam para a superação da situação existencial. Este modelo considera que o sujeito deve compartilhar e ser parceiro dos projetos e processos e, que é por meio dos espaços relacionais que ele restaura sua contratualidade de cidadão e de produtor de sentido para sua vida (Mângia, 2002).

Apesar do movimento de transformação da assistência, as pessoas com transtornos mentais no Brasil ainda têm sido marginalizadas e excluídas socialmente. Nesta perspectiva, surgem as práticas pautadas pelo paradigma social de reabilitação que visam a inclusão social, isto é, a construção de espaços sociais receptivos para atender populações com algum tipo de diferença ou deficiência, e sujeitos com o desejo de ocupar um lugar de participação na vida social (Ghirardi, 1999).

Os "Centros de Convivência e Cooperativas" (CeCCOs) adotados pela prefeitura de São Paulo, no período de 1989 a 2000, foram baseados neste paradigma. Estes equipamentos propunham a convivência entre pessoas portadoras de transtorno mental, portadores de deficiência física e/ou sensorial, idosos, crianças e adolescentes em situação de risco pessoal e social, e a população em geral em espaços públicos, tais como, parques, praças, centros comunitários. Como estratégia de ação utilizavam-se de oficinas nas quais se desenvolviam vários tipos de atividades, partindo-se das necessidades dos usuários, valorizando-se sua história e sua identidade sócio-cultural (Lopes \& Leão, 2002).

Ao analisar a participação de terapeutas ocupacionais nos CeCCos, os autores referem que a terapia ocupacional tem um espaço importante nestes equipamentos porque "os fundamentos da profissão vêm ao encontro da proposta de produzir e conceber saúde e, principalmente, pelo uso do recurso "atividade" para a busca de autonomia e da participação social" (Lopes \& Leão, 2002, p.62).

Como exemplo de uma prática da terapia ocupacional promotora de estratégias de inclusão social, pode-se citar a experiência da Associação Arte e Convívio (AAC), no município de Botucatu-SP, tema de uma dissertação de mestrado (Ribeiro, 2003). Esta associação da área de saúde mental foi criada em 1995, a partir da iniciativa de profissionais, pacientes e pessoas da comunidade, diante da falta de oficinas terapêuticas e de convivência na rede pública de assistência. Embora tivesse contado com participação multiprofissional, a estruturação desta Associação fundamentou-se em pressupostos e formas de atuação da terapia ocupacional.

A AAC teve como ponto de partida o tratamento clínico dos pacientes nos serviços de saúde mental. Neste processo observava-se que embora os pacientes estivessem fora dos hospitais psiquiátricos, viviam como se estivessem internados, isolados em suas casas, sem executar atividades nem mesmo de lazer. Não tinham participação social nem política e enfrentavam diversas dificuldades para se inserirem no mercado de trabalho. Viviam numa rotina pobre e sem sentido. $O$ reconhecimento do sofrimento causado pela situação existencial dos pacientes foi o grande mobilizador do projeto.

As estratégias que a AAC utiliza para lidar com estas questões são: a criação de espaços de convivência entre usuários (adultos e adolescentes), familiares, trabalhadores dos serviços de saúde mental e pessoas da comunidade; a realização de oficinas terapêuticas, de geração de renda e de relaxamento; a organização de festas, viagens, passeios e eventos científicos, entre outras.

Os produtos das oficinas de geração de renda são 
comercializados e parte do dinheiro arrecadado é destinada aos usuários. Além disto, os profissionais procuram exercer uma prática de cuidado horizontalizada, estimulando os usuários a compartilharem e serem parceiros dos projetos e decisões, bem como a se conscientizarem de seus direitos de cidadãos.

A AAC tem desenvolvido uma práxis que rompe com o modelo asilar excludente de tratar as pessoas com transtorno mental, envolvendo a sociedade desde a fase de sua construção ideológica. Tem sido um lugar de possibilidade de existência criativa para os usuários e conseguido a inclusão de alguns no mercado de trabalho (Ribeiro, 2003).

\section{Considerações finais}

Com a implantação da rede de serviços substitutivos, o terapeuta ocupacional passa a ter uma prática voltada para atender os pacientes graves fora da internação psiquiátrica. Diferente da ação periférica e desqualificada desenvolvida no hospital, cuja atuação era basicamente ocupá-los para manter a ordem, com pouca interferência na promoção de saúde e na melhora da condição de vida dos mesmos (Mângia, s/d, apud Terapia, 2004).

Segundo Medeiros (2003), o instrumental da profissão mostra-se condizente com as proposições da transformação assistencial atual, uma vez que o usuário dos serviços passa a ser encarado como um indivíduo que se realiza e restabelece sua saúde mediante sua (re)inclusão social. Desta forma, o paradigma utilizado não poderá ser somente o biológico centrado na doença e nos sintomas, independente do contexto, mas sim aquele que busca atender o usuário em suas necessidades e com toda a complexidade da sua condição socioeconômica e cultural.

Por ser uma profissão que congrega conhecimentos de várias disciplinas, a terapia ocupacional pode "ser um elemento importante na construção de novos rumos para a atenção à saúde, integral, globalizante e na perspectiva da totalidade, subjetividade e singularidade das pessoas" (Medeiros 2003, p.173). Neste sentido, os terapeutas ocupacionais que buscam um aparato teórico e técnico para o tratamento e para se ocuparem das dificuldades e necessidades dos pacientes, procurando reinventar o cotidiano deles e atender aos interesses sociais, políticos e à saúde pública, abrem com isto, um campo interessante, bastante ampliado e valorizado de atuação da profissão na comunidade.

\section{Referências}

AMARANTE, P. Loucos pela vida. 2.ed. Rio de Janeiro: Panorama, ENSP, 2000.

ARAUJO, A. O acompanhamento terapêutico no processo de reabilitação psicossocial de pacientes psiquiátricos com longa história de internação. 1999. Dissertação (Mestrado) - Universidade Federal de São Carlos, São Carlos.

BASAGLIA, F. A instituição negada: relato de um hospital psiquiátrico. Rio de Janeiro: Graal, 1991.

BRASIL. Ministério da Saúde. Conselho Nacional de Saúde. III Conferência Nacional de saúde mental: relatório final. Brasília, 2002.

CESARINO, A. C. Uma experiência de saúde mental na prefeitura de São Paulo. In: LANCETTI, A. 
(Org.) Saudeloucura1. São Paulo: Hucitec, 1989. p.3-30.

GHIRARDI, M. I. G. Representações da deficiência e prática de reabilitação: uma análise do discurso técnico. 1999. Tese (Doutorado) - Faculdade de Psicologia, Universidade de São Paulo, São Paulo.

LOPES, R. E.; LEÃO, A. Terapeutas ocupacionais e os centros de convivência e cooperativas: novas ações de saúde. Rev. Ter. Ocup. Univ. São Paulo, v.13, n.2, p.56-63, 2002.

LUSSI, I. A. O. Concepção sistêmica do indivíduo: auto-organização e reabilitação psicossocial. 2003. Dissertação (Mestrado) - Faculdade de Filosofia e Ciências, Universidade Estadual Paulista, Marília.

MÂNGIA, E. F. Contribuições da abordagem canadense "prática de Terapia Ocupacional centrada no cliente" e dos autores da desinstitucionalização italiana para a Terapia Ocupacional em saúde mental. Rev. Ter. Ocup. Univ. São Paulo, v.13, n.3, p.127-34, 2002.

MEDEIROS, M. H. R. Terapia Ocupacional: um enfoque epistemológico e social. São Paulo: Hucitec, 2003.

PITTA, A. M. F. Sobre uma política de saúde mental. 1984. Dissertação (Mestrado) Universidade de São Paulo, São Paulo.

RESENDE, H. Política de saúde mental no Brasil: uma visão histórica. In: TUNDIS, S. A.; COSTA, N. R. (Orgs.) Cidadania e loucura: políticas de saúde mental no Brasil. Rio de Janeiro: Vozes/ ABRASCO, 1987. p.15-74.

RIBEIRO, M. B. S. Estudo de características familiares de usuários de uma associação civil para a reabilitação psicossocial. 2003. Dissertação (Mestrado) - Faculdade de Medicina de Botucatu, Universidade Estadual Paulista, Botucatu.

ROTELLI, F.; LEONARDIS, O.; MAURI, D. Desinstitucionalização, uma outra via: a reforma psiquiátrica italiana no contexto da Europa e dos "Países Avançados". In: NICÁCIO, F. (Org.) Desinstitucionalização. São Paulo: Hucitec, 2001. p.17-59.

SARACENO, B. Libertando identidades: da reabilitação psicossocial à cidadania possível. 2.ed. Rio de Janeiro: Te Cora/Instituto Franco Basaglia, 2001.

SILVA FILHO, J. F. A medicina, a psiquiatria e a doença mental. In: TUNDIS, S. A.C.; COSTA, N. R. Cidadania e loucura: políticas de saúde mental no Brasil. Rio de Janeiro: Vozes/ABRASCO, 1987. p.75-102.

TERAPIA ocupacional analisa a reforma psiquiátrica. Coffito, n.3 (textos isolados). Disponível em: <http//www.coffito.org.br>. Acesso em: 03 jun. 2004.

YASUI, S. A. A construção da reforma psiquiátrica e o seu contexto histórico. 1999. Dissertação (Mestrado) - Faculdade de Ciências e Letras, Universidade Estadual Paulista, Assis. 


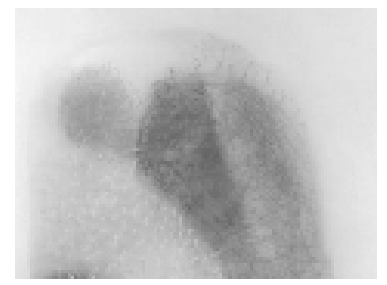

Since its inception, Brazilian psychiatric care has been based on interning the mentally ill in psychiatric hospitals and on the social exclusion of patients. Ever since the end of the military regime in the eighties, this assistance has been undergoing a transformation that proposes the treatment of the mentally ill by means of community services, replacing psychiatric hospitals. The occupational therapy profession, whose practice used to focus on the occupation of patients within hospitals, in the light of the transformation of psychiatric care, has sought theoretical, technical and political development for working in these substitutive services, in terms of prevention, health promotion, treatment, rehabilitation and social inclusion. The objective of this article is to present certain occupational therapy practices based on paradigms that emphasize the importance of treatment and inclusion of the mentally ill in society, highlighting an experience that is currently being carried out in Botucatu, São Paulo State, Brazil, by a non-governmental organization. It was possible to conclude that the profession, in uniting interdisciplinary understanding and in concerning itself with the needs and difficulties of patients' daily lives, presents an instrument conducive to community assistance. KEY WORDS: Psychiatric care; mental health; social inclusion; occupational therapy.

A assistência psiquiátrica brasileira, desde seu início, era baseada na internação dos doentes mentais em hospitais psiquiátricos e em sua exclusão social. Desde o final do regime militar, na década de oitenta, esta assistência vem passando por transformações que propõem o tratamento dos doentes mentais em serviços comunitários substitutivos ao hospital psiquiátrico. A profissão terapia ocupacional cuja prática voltava-se para a ocupação dos pacientes no interior dos hospitais, diante das transformações da assistência psiquiátrica, vem buscando um aprimoramento teórico técnico e político para a atuação nos serviços substitutivos, em nível de prevenção, promoção de saúde, tratamento, reabilitação e inclusão social. O presente trabalho tem como objetivo apresentar algumas práticas de terapia ocupacional baseadas em paradigmas que enfatizam a importância do tratamento e da inclusão do doente mental na sociedade, destacando-se uma experiência que vem sendo realizada em Botucatu-SP (Brasil), por uma organização não governamental. Conclui-se que a profissão, por congregar conhecimento interdisciplinar, e se ocupar das necessidades e dificuldades dos pacientes no cotidiano, apresenta um instrumental condizente com a assistência comunitária.

PALAVRAS-CHAVE: Assistência psiquiátrica; saúde mental; inclusão social; terapia ocupacional.

La asistencia psiquiátrica brasileña, desde su inicio, se basaba en la internación de los enfermos mentales en hospitales psiquiátricos y en su exclusión social. Desde el final del régimen militar, en la década de los ochenta, esta asistencia viene sufriendo transformaciones que proponen el tratamiento de los enfermos mentales en servicios comunitarios sustitutivos del hospital psiquiátrico. Ante las transformaciones de la asistencia psiquiátrica, la profesión Terapia Ocupacional, cuya práctica se abocaba a la ocupación de los pacientes en el interior de los hospitales, está empeñada en perfeccionarse teórica, técnica y políticamente para la actuación en los servicios sustitutivos, en las áreas de prevención, promoción de la salud, tratamiento, rehabilitación e inclusión social. El presente trabajo tiene como objetivo presentar algunas prácticas de terapia ocupacional fundamentadas en paradigmas que enfatizan la importancia del tratamiento y de la inclusión del enfermo mental en la sociedad, y destaca una experiencia que viene siendo realizada en Botucatu-SP (Brasil), por una organización no gubernamental. Se concluye que la profesión, por congregar conocimiento interdisciplinario y ocuparse de las necesidades y dificultades cotidianas de los pacientes, presenta un instrumento apropiado para la asistencia comunitaria.

PALABRAS CLAVE: Asistencia psiquiátrica; salud mental; inclusión social; terapia ocupacional. 


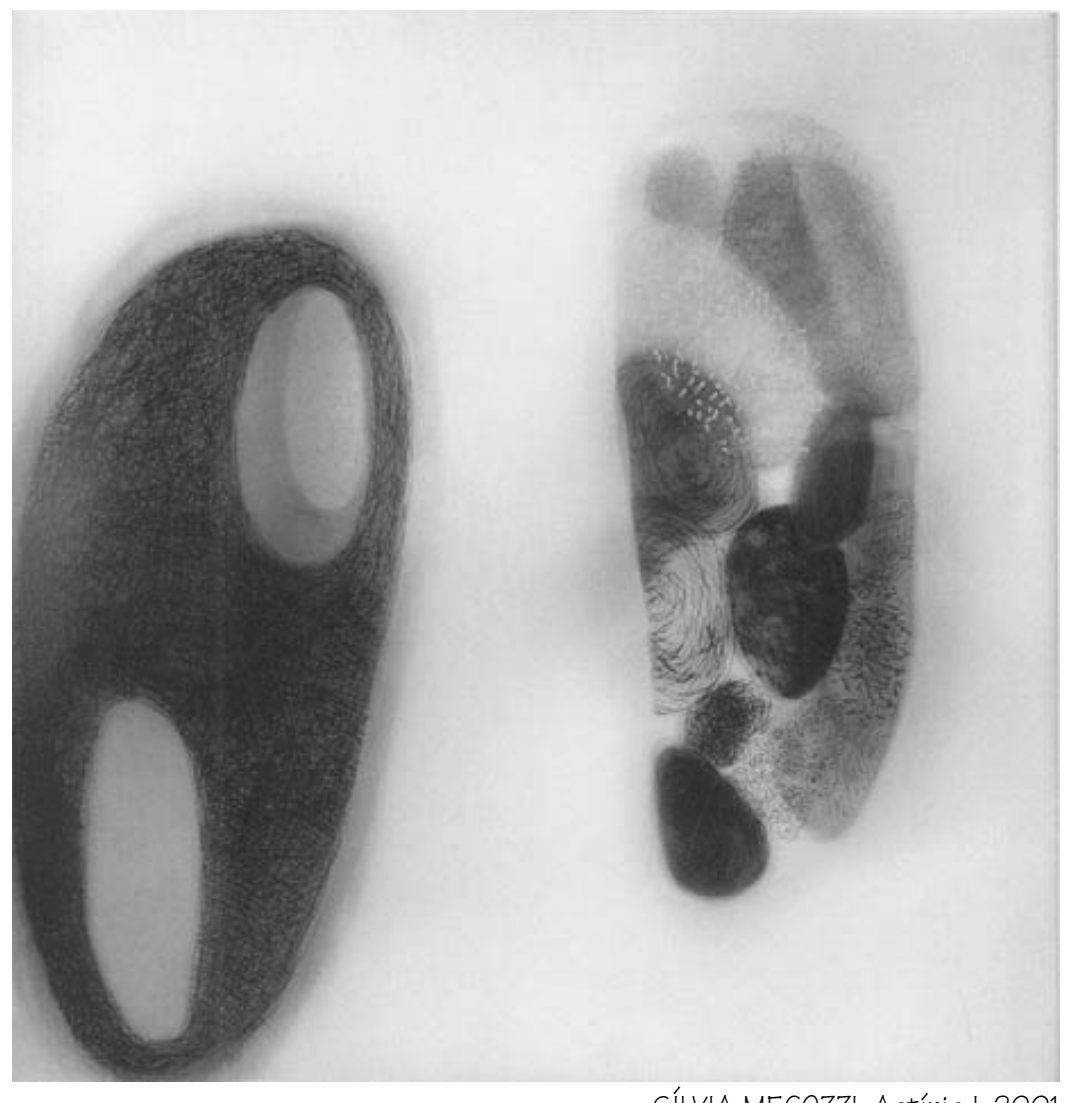

SÍLVIA MECOZZI, Actínia I, 2001

432 Interface - Comunic, Saúde, Educ, v.9, n.17, p.432, mar/ago 2005 